[SPECIAL EDITION on Annual Meeting and Symposium 2017-Transaction】

\title{
Synchrotron SAXS Studies on Lattice Structure of Spherical Micelles in Binary Mixtures of Block Copolymers and Homopolymers
}

\author{
Hideaki Takagi*1,\#1 , Ryo Hashimoto*1, Noriyuki Igarashi*1, \\ Shunji Kishimoto*1, and Katsuhiro Yamamoto*2.\#2 \\ ${ }^{*}$ Photon Factory, Institute of Materials Structure Science, High Energy Accelerator Research Organization, 1-1 Oho, \\ Tsukuba, Ibaraki 305-0801, Japan \\ ${ }^{* 2}$ Graduate School of Engineering, Department of Life Science \& Applied Chemistry, Nagoya Institute of Technology, \\ Gokiso-cho, Showa-ku, Nagoya 466-8555, Japan
}

\begin{abstract}
The lattice structures of spherical micelles in binary mixtures of polybutadiene-poly( $(\varepsilon$-caprolactone) diblock copolymers and polybutadiene homopolymers were investigated using synchrotron small angle X-ray scattering technique. In the blend sample with a weight fraction of added homopolymer ( $\left.\phi_{\text {homo }}\right)$ of 40 wt\%, hexagonally packed cylinders were formed, while spherical morphologies were arrayed into the Frank-Kasper $\sigma$ phase at $\phi_{\text {homo }}=50 \mathrm{wt} \%$. Closely packed spheres in hexagonal closely packed (HCP) and face-centered cubic (FCC) lattices were observed for the sample with $\phi_{\text {homo }}=56$ and $67 \mathrm{wt} \%$, respectively. It was found that the

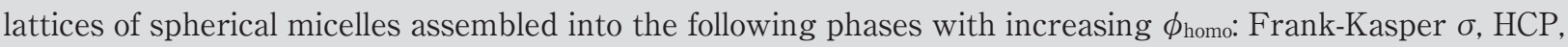
and FCC phases. (Received 28 July, 2017; Accepted 29 September, 2017)
\end{abstract}

\section{Introduction}

When the compositional asymmetry in a block copolymer increases significantly, block copolymer self-assembly results in the formation of spherical morphologies [1]. In general, spheres composed of block copolymers are arranged in body-centered cubic (BCC) lattice in many block copolymer systems. In a simple diblock copolymer system, it is predicted that the closely packed spheres in hexagonal closely packed (HCP) and face-centered cubic (FCC) lattices exist outside as an ordered phase in the phase diagram [2], and the subsequent experiments confirmed that the FCC phase was found in a diblock copolymer [3].

Recently, it was reported that spherical micelles could be arrayed in a variety of lattices [4]. For example, in a polystyrene-polyisoprene-polystyrenepoly(ethylene oxide) linear tetrablock copolymer (PSPI-PS-PEO), the Frank-Kasper $\sigma$ phase and the associated dodecagonal quasicrystal were observed
$[5,6]$. The $\sigma$ phase is often found in close proximity to quasicrystals, and contains 30 particles in the unit cell with $P 4_{2} / m n m$ space group symmetry. Recent experiments revealed the formation of the FrankKasper A 15 phase (space group $P m 3 n$ ) in PS-PI-PSPEO [7]. Various types of lattices were also seen in simple diblock copolymer systems. The Frank-Kasper $\sigma$ phase and the dodecagonal quasicrystal were observed in a polyisoprene-polylactide diblock copolymer (PI-PLA) [5,8,9]. Most recently, FrankKasper C 14 and C 15 Laves phases were also discovered in PI-PLA [10]. Note that it was reported that the dodecagonal quasicrystals observed in diblock copolymers were in a non-equilibrium state and transformed into the stable phase after annealing over a long period of time [9].

Such well-defined nanostructures have been studied for application as optical materials [11]. In particular, photonic crystals attract increasing interest in the field of nanotechnology [4,12-15]. Some research groups reported photonic crystals composed

\#1 corresponding author: Hideaki Takagi (E-mail: takagih@post.kek.jp)

\#2 corresponding author: Katsuhiro Yamamoto (E-mail: yamamoto.katsuhiro@nitech.ac.jp) 
of soft materials, such as colloids [13], high-molecularweight block copolymers [14], and block copolymer solutions [15]. Quasiperiodic structures can induce and widen the photonic band gap easily due to their high symmetry, prohibiting light within a range of wavelengths from propagating in any direction.

The region within which the Frank-Kasper $\sigma$ phase exists in a phase diagram has also been studied. The self-consistent field theory (SCFT) calculations demonstrated that the $\sigma$ phase becomes stable in the region between hexagonally packed cylinders (HEX) and BCC on a phase diagram, and the thermodynamical stable range of the $\sigma$ phase extends with increasing conformational asymmetry $\varepsilon^{2}=v_{\mathrm{A}} a_{\mathrm{A}}{ }^{2} /$ $\left(v_{\mathrm{B}} a_{\mathrm{B}}{ }^{2}\right)$, where $v$ and $a$ are the molecular volume and the statistical segmental length, respectively [16]. With the increase in $\varepsilon$, spherical micelles are transformed into polyhedral shapes, and thus, polyhedral particles can form complex lattices. A recent experimental study elucidated that the $\sigma$ phase was not observed in poly ( ethylene-alt - propylene ) - polylactide diblock copolymer with $\varepsilon=1.06$, while the $\sigma$ phase appeared as a stable phase in modestly asymmetric polyisoprene-polylactide diblock copolymer $(\varepsilon=1.32$ ); increasing the conformational asymmetry to $\varepsilon=1.68$ (polyethylethylene-polylactide diblock copolymer) broadened the $\sigma$ phase region, which was consistent with SCFT calculation results [17].

Our research group has investigated the phase behavior of a binary mixture of a block copolymer and a homopolymer. Recently, the Frank-Kasper $\sigma$ phase was observed in polybutadiene-poly( $\varepsilon$-caprolactone) (PB-PCL) diblock copolymer/polybutadiene (PB) blends [18]. In this study, we investigated the type of lattice structure formed depending on the amount of the added homopolymers in spherical-morphologyforming block copolymer/homopolymer blends. It was found that the lattice changed in the following order with increasing amount of homopolymers: the Frank-Kasper $\sigma$, HCP, and FCC phases.

\section{Experimental section}

\subsection{Materials}

PB-PCL and PB were synthesized by living anionic polymerization [18]. The number-average molecular weight $\left(M_{\mathrm{n}}\right)$ and its heterogeneous index $\left(M_{\mathrm{w}} / M_{\mathrm{n}}\right)$ were determined by size-exclusion chromatography (SEC) using PB standards. The volume fraction of PCL $\left(f_{\mathrm{PCL}}\right)$ was calculated using
Table 1 Molecular characteristics of PB-PCL and PB.

\begin{tabular}{lccc}
\hline & $M_{\mathrm{n}}{ }^{\mathrm{a}}$ & $M_{\mathrm{w}} / M_{\mathrm{n}}{ }^{\mathrm{a}}$ & $f_{\mathrm{PCL}}(\text { vol. } \%)^{\mathrm{b}}$ \\
\hline PB-PCL & $7600^{\mathrm{c}}$ & 1.08 & 41 \\
\hline PB & 900 & 1.1 & - \\
\hline${ }^{\mathrm{a}}$ Determined by SEC using PB standards. \\
${ }^{\mathrm{b}}$ Determined by ${ }^{1} \mathrm{H}-\mathrm{NMR}$. \\
${ }^{\mathrm{c}}$ Determined by SEC and ${ }^{1} \mathrm{H}-\mathrm{NMR}$.
\end{tabular}

proton nuclear magnetic resonance $\left({ }^{1} \mathrm{H}-\mathrm{NMR}\right)$ spectroscopy. The parent PB-PCL shows a crystalline-amorphous alternating lamellar structure in the crystalline state and a lamellar structure in molten state $\left(T>60^{\circ} \mathrm{C}\right)$. The molecular characteristics of PB-PCL and PB are presented in Table 1. The blend samples were prepared according to the standard method [18].

\subsection{Small angle X-ray scattering}

Synchrotron small angle X-ray scattering (SAXS) measurements were conducted at the beamline BL-6 A [19] and BL-15A2 [20] in Photon Factory (PF) of High Energy Accelerator Research Organization in Tsukuba, Japan (KEK). The detector was PILATUS3 1M (Dectris) in BL-6A. In BL-15A2, PILATUS3 2M and a silicon-on-insulator photon imaging array sensor (SOPHIAS) detectors were used. The pixel size of SOPHIAS detector is $30 \mu \mathrm{m}$, which is smaller than that of PILATUS3 $(172 \mu \mathrm{m})$ [21]. The X-ray energy was $8.3 \mathrm{keV}$, and the sample-detector distance was $2.5 \mathrm{~m}$ in BL-6 A experiments. In BL-15A2 experiments, the X-ray energy was $10.2 \mathrm{keV}$, and the sampledetector distance was $3.5 \mathrm{~m}$. Silver behenate and collagen (chicken tendon) were used as the standard specimens to calibrate SAXS detector. The scattering intensities were corrected for background scattering and sample absorption. The magnitude of the scattering vector $(q)$ is given by

$$
q=\frac{4 \pi}{\lambda} \sin \theta
$$

where $\lambda$ is the wavelength of the X-ray and $2 \theta$ is the scattering angle. The sample temperature was controlled using Linkam LK-600 M equipment (Japan Hightech). In the SAXS experiments, the sample was heated to a target temperature at a rate of $100{ }^{\circ} \mathrm{C} / \mathrm{min}$. After the sample was held for $600 \mathrm{~s}$ at the target temperature, the scattering data were recorded. Just before recording the data, X-ray irradiation was not performed to avoid X-ray damage to the sample. The temperature was raised from $60{ }^{\circ} \mathrm{C}$ to $220{ }^{\circ} \mathrm{C}$ in increments of $20{ }^{\circ} \mathrm{C}$. It has been previously discussed that the thermal degradation of copolymer chains 
occurred at high temperatures [7,22]. In this study, the total time above $220{ }^{\circ} \mathrm{C}$ is only ca. 20 min, meaning that the amount of thermally degraded copolymer chains should be relatively small. In addition, thermoreversibility of SAXS patterns was also confirmed. Thus, it was considered that there was no effect of thermal degradation on the phase behavior. Data processing was conducted using SAngler software [23].

\section{Results and discussion}

SEC charts of synthesized PB-PCL (solid line) and PB (dashed line) are shown in Fig. 1. SEC charts for both PB-PCL and PB show a monomodal and symmetric peak $\left(M_{\mathrm{w}} / M_{\mathrm{n}} \leq 1.1\right)$.

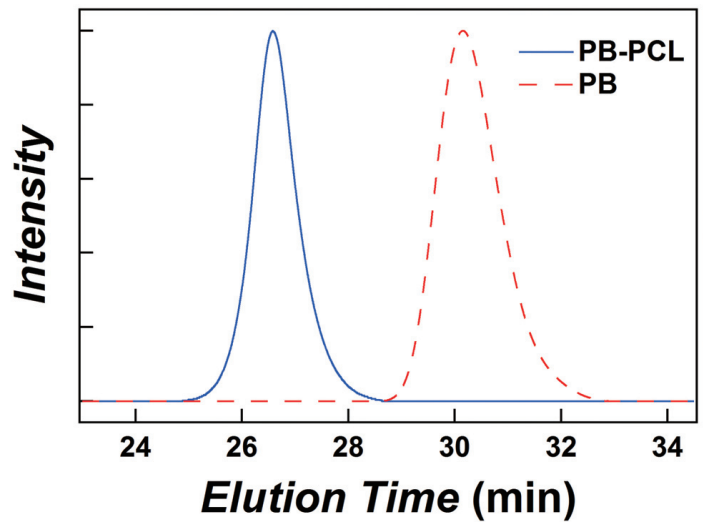

Fig. 1 SEC elution curves for synthesized polymers. The solid line represents PB-PCL, and the dashed line corresponds to $\mathrm{PB}$.

Fig. 2 displays SAXS profile for the PB-PCL/PB blend obtained at $200{ }^{\circ} \mathrm{C}$. The weight fraction of added homopolymer $\left(\phi_{\text {homo }}\right)$ was $40 \mathrm{wt} \%$. Data are plotted as the logarithm of the relative scattered intensity log $I(q)$ versus the magnitude of the scattering vector $q$. The sharp scattering peaks are clearly seen at the relative $q$-peak position of $1: 3^{1 / 2}: 7^{1 / 2}: 9^{1 / 2}$ as marked by arrows. This result indicates that HEX were formed. Generally, the diffraction peaks of HEX can be observed at the relative $q$-peak position of $1: 3^{1 / 2}: 4^{1 / 2}: 7^{1 / 2}$ : $9^{1 / 2}$. The lack of the $4^{1 / 2}$ peak should be attributed to the superposition of the peak position of a hexagonal structural factor and the valley position of a cylindrical form factor. Theoretically, the $4^{1 / 2}$ peak almost disappears when the volume fraction is around $27.4 \mathrm{vol} \%$ [24]. In the blend sample with $\phi_{\text {homo }}=40 \mathrm{wt} \%$, the overall volume fraction of PCL is $26.6 \mathrm{vol} \%$, which is almost consistent with the theoretical extinction condition. Thus, the $4^{1 / 2}$ peak was not observed in this sample.

The SAXS profile for the blend sample with $\phi_{\text {homo }}$

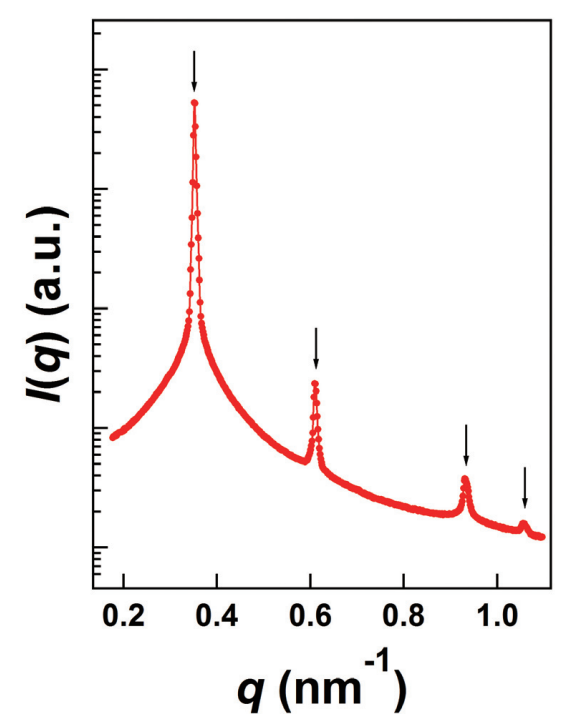

Fig. 2 SAXS profile of the PB-PCL/PB blend $\left(\phi_{\mathrm{PB}}=\right.$ $40 \mathrm{wt} \%)$ obtained at $200{ }^{\circ} \mathrm{C}$. Arrows indicate scattering peaks of HEX.

$=50 \mathrm{wt} \%$ obtained at $220{ }^{\circ} \mathrm{C}$ is shown in Fig. 3 (a). Fig. 3 (b) displays the enlarged SAXS profile around $0.25 \leq q \leq 0.38 \mathrm{~nm}^{-1}$ using SOPHIAS detector, which has the advantage of smaller pixel sizes $(30 \mu \mathrm{m})$ than PILATUS 3 detector $(172 \mu \mathrm{m})$. Arrows in Figs. 3 (a) and (b) represent the peak positions, while the numbers in brackets represent the reflection indexes of the Frank-Kasper $\sigma$ phase ( $\mathrm{P}_{2} / \mathrm{mnm}$ space group) [18]. The calculated and observed scattering peak positions in $0.23 \leq q \leq 0.4 \mathrm{~nm}^{-1}$ are listed in Table 2. It is easily seen in Figs. 3 (a) and (b) and Table 2 that all of the observed scattering peaks are in good agreement with calculated ones. In addition, the value of the lattice parameter ratio of $a$-axis $/ c$-axis is 1.90 ( $a$-axis $=$ $85.9 \mathrm{~nm}$ and $c$-axis $=45.3 \mathrm{~nm}$ ). This value is almost coincident with values obtained in previous studies $[4,18]$. Therefore, it was found that the Frank-Kasper $\sigma$ phase was observed at $220^{\circ} \mathrm{C}$.

Fig. 4 displays the SAXS profile for the sample with $\phi_{\text {homo }}=56 \mathrm{wt} \%$ obtained at $200{ }^{\circ} \mathrm{C}$. The scattering peaks are observed at the relative peak position of 1:1.06:1.13:1.43:1.72:1.86:2.06:2.69. The calculated relative $q$-peak positions and the reflection indexes for HCP and FCC phases are listed in Table 3. The series of observed scattering peaks are correspond very well with the HCP lattice structure. Here, to compare the experimental SAXS profiles with the theoretical ones, the theoretical scattering curves were calculated [25]. The scattering intensity can be expressed as

$$
I(q)=\left(b_{1}-b_{2}\right)^{2} \rho_{N} P(q) S(q)
$$



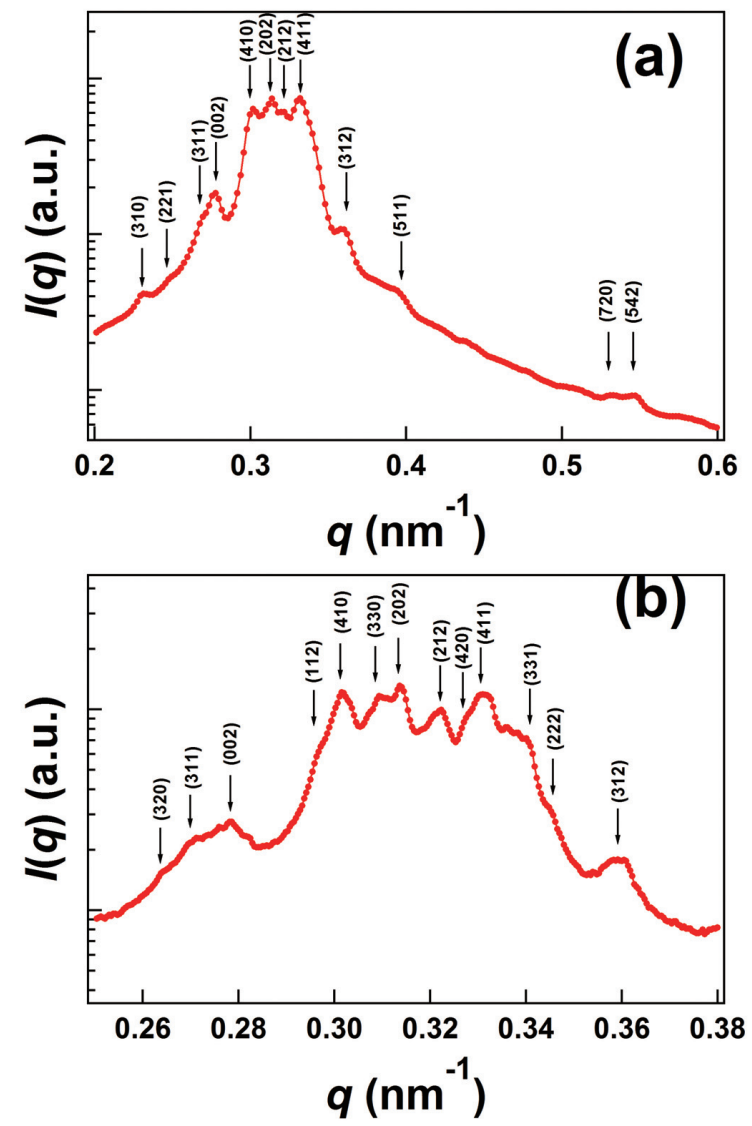

Fig. 3 (a) SAXS profile of the PB-PCL/PB blend $\left(\phi_{\mathrm{PB}}=50 \mathrm{wt} \%\right)$ obtained at $220{ }^{\circ} \mathrm{C}$ and (b) the SAXS profile obtained using SOPHIAS detector (see text). Arrows indicate scattering peaks and the numbers in brackets mean the reflection indexes of the Frank-Kasper $\sigma$ phase.

Table 2 Calculated and observed peak positions of the $\sigma$ phase.

\begin{tabular}{ccc}
\hline$h k l$ & $q_{\text {calc. }}$ & $q_{\text {obs. }}$ \\
\hline 310 & 0.231 & 0.231 \\
\hline 221 & 0.249 & 0.247 \\
\hline 301 & 0.259 & - \\
\hline 320 & 0.264 & 0.263 \\
\hline 311 & 0.27 & 0.269 \\
\hline 200 & 0.278 & 0.278 \\
\hline 400 & 0.293 & - \\
\hline 112 & 0.296 & 0.296 \\
\hline 321 & 0.298 & - \\
\hline 410 & 0.302 & 0.302 \\
\hline 330 & 0.311 & 0.309 \\
\hline 202 & 0.313 & 0.314 \\
\hline 212 & 0.322 & 0.322 \\
\hline 420 & 0.327 & 0.328 \\
\hline 411 & 0.332 & 0.332 \\
\hline 331 & 0.34 & 0.339 \\
\hline 222 & 0.346 & 0.345 \\
\hline 421 & 0.355 & - \\
\hline 312 & 0.361 & 0.360 \\
\hline 430 & 0.365 & - \\
\hline 510 & 0.373 & - \\
\hline 322 & 0.383 & - \\
\hline 501 & 0.392 & - \\
\hline 520 & 0.394 & - \\
\hline 511 & 0.398 & 0.398 \\
\hline & & \\
\hline & & \\
\hline 323
\end{tabular}

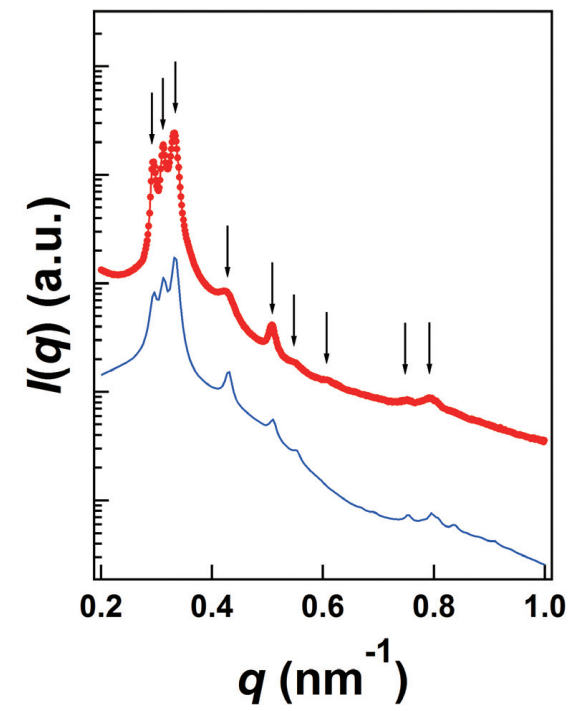

Fig. 4 SAXS profile of the PB-PCL/PB blend $\left(\phi_{\mathrm{PB}}=\right.$ $56 \mathrm{wt} \%)$ obtained at $200{ }^{\circ} \mathrm{C}$. The solid line shows the calculated SAXS curve obtained using eq. (2). Arrows represent HCP diffraction peaks.

$b_{1}$ and $b_{2}$ are scattering length densities of the particles and the surrounding matrix, respectively, and $\rho_{\mathrm{N}}$ is the number density of the particles. $P(q)$ is the form factor and $S(q)$ is the structural factor. $S(q)$ is given by

$$
S(q)=1+\beta(q)[Z(q)-1] G(q)
$$

$\beta(q)=\langle F(q)\rangle /\left\langle F^{2}(q)\right\rangle$ is the ratio of the squared scattering amplitude, $G(q)$ is the Debye-Waller factor, and $Z(q)$ is the lattice factor. The spherical micelles with radius $R$ was used as $P(q)$

$$
P(q)=\left[\frac{3(\sin (q R)-q R \cos (q R))}{(q R)^{3}}\right]^{2}
$$

The size distribution of $P(q)$ was modeled using a Shultz-Zimm distribution. The solid line in Fig. 4 indicates the calculated SAXS profile. The calculated profile is also in excellent agreement with the experimental one. Consequently, spherical micelles are arranged in the HCP lattice.

Fig. 5 presents the SAXS profile for the blend with $\phi_{\text {homo }}=67 \mathrm{wt} \%$ obtained at $160{ }^{\circ} \mathrm{C}$. The relative $q$-peak position of 1:1.15:1.61:1.91:2.53 is observed. As shown in Table 3 , these scattering peak positions agree with those of the FCC lattice structure. Moreover, the calculated scattering curve for the FCC lattice structure using eq. (2) is also shown in Fig. 5 (solid line). The dash line represents the form factor obtained using eq. (4). Both experimental and theoretical profiles seem to resemble each other, 
Table 3 Reflection indexes and relative peak positions of the lattice of FCC and HCP.

\begin{tabular}{cccc}
\hline \multicolumn{2}{c}{ FCC } & \multicolumn{2}{c}{ HCP } \\
\hline $\begin{array}{c}\text { Reflectio } \\
\text { n Index } \\
(h k l)\end{array}$ & $\begin{array}{c}\text { Relative } \\
\text { position }\end{array}$ & $\begin{array}{c}\text { Reflectio } \\
\text { n Index } \\
(h k l)\end{array}$ & $\begin{array}{c}\text { Relative } \\
\text { position }\end{array}$ \\
\hline$(111)$ & 1 & $(100)$ & 1 \\
\hline$(200)$ & 1.15 & $(002)$ & 1.06 \\
\hline$(220)$ & 1.63 & $(101)$ & 1.13 \\
\hline$(311)$ & 1.91 & $(102)$ & 1.46 \\
\hline$(222)$ & 2 & $(110)$ & 1.73 \\
\hline$(400)$ & 2.31 & $(103)$ & 1.86 \\
\hline$(331)$ & 2.52 & $(112)$ & 2.04 \\
\hline & & $(201)$ & 2.07 \\
\hline & & $(004)$ & 2.12 \\
\hline & $(202)$ & 2.26 \\
\hline & $(104)$ & 2.34 \\
\hline & $(203)$ & 2.56 \\
\hline & $(210)$ & 2.65 \\
\hline
\end{tabular}

indicating that the lattice structure is FCC.

Here, we calculated the sphere radius $(R)$ in each blend sample using the scattering peak position and the volume fraction of PCL. The radii in the FCC, HCP, and the $\sigma$ phases are given respectively by

$$
\begin{aligned}
& R_{F C C}=\sqrt{3}\left(\frac{3 f_{c}}{16 \pi}\right)^{1 / 3} d_{111} \\
& R_{H C P}=\frac{2}{\sqrt{3}}\left(\frac{3 \sqrt{2} f_{c}}{8 \pi}\right)^{1 / 3} d_{100} \\
& R_{\sigma}=\left(\frac{2 \sqrt{17} f_{c} d_{002}{ }^{2} d_{410}}{40 \pi}\right)^{1 / 3}
\end{aligned}
$$

where $f_{\mathrm{c}}$ is a volume fraction of PCL and $d_{\mathrm{hkl}}$ is a domain spacing of $(h k l)$ plane. We obtained $R_{\mathrm{FCC}}=$ $7.8 \mathrm{~nm}, R_{\text {HСP }}=8.0 \mathrm{~nm}$, and $R_{\sigma}=8.4 \mathrm{~nm}$. In addition, the first maximum peak position of the spherical form factor is seen in Fig. 5 marked by the thick arrow. $R$ can be also calculated from the maximum peak position of the form factor. The spherical morphology is given by $q^{*} R=5.765$, where $q^{*}$ is the scattering maximum value of the spherical form factor. $R$ calculated from the form factor is $8.1 \mathrm{~nm}$. Thus, the radius of spherical micelles does not change regardless of the amount of homopolymers.

Next, the change in the lattice structure of spherical micelles depending on the amount of added homopolymers is studied. Theoretical studies demonstrate that the Frank-Kasper $\sigma$ phase, which is the most complicated lattice observed in this study, can become stable only when the conformational asymmetry is relatively large [16]. The spherical

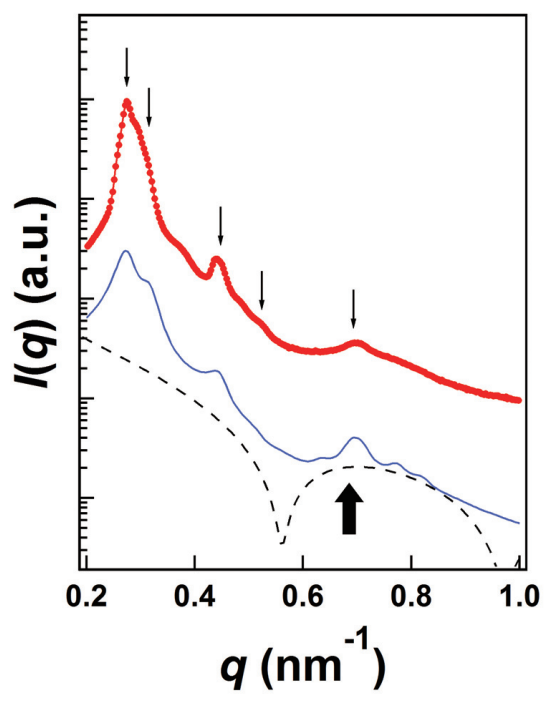

Fig. 5 SAXS profiles of the PB-PCL/PB blend $\left(\phi_{\mathrm{PB}}=\right.$ $67 \mathrm{wt} \%$ ) obtained at $160{ }^{\circ} \mathrm{C}$ and the calculated SAXS profile obtained using eq. (2) (solid line). The dash line shows the form factor calculated using eq. (4). Thin arrows show FCC diffraction scattering peaks, and the thick arrow shows the maximum value of the form factor.

surface of micelles is deformed to polyhedral shapes with the increase in $\varepsilon$, leading to the formation of the complicated lattices. Since $\varepsilon_{\text {PB.PCL }}$ is determined to be 1.55 , which is relatively larger than the critical value ( $\varepsilon=1.5)$ [18], the $\sigma$ phase emerges as a stable phase in the blend sample.

On the other hand, because the region corresponding to FCC and HCP phases in the theoretical phase diagram hardly changes with the increase in $\varepsilon$ [16], there are other reasons of the observed HCP and FCC phases. It is well known that the differences in the free energy between $\mathrm{FCC}$ and HCP phases in a hard sphere model is very small, and the FCC phase is only slightly more stable than the HCP phase [26]. It was also found that the HCP phase cannot be stable in case of weakly charged hard spheres [27] and charged hard spheres [28]. Moreover, theoretical study results predict that elastic spheres are never arrayed into only the HCP lattice [29]. The FCC lattice structure was found mainly in soft matter systems, but the HCP lattice structure was only rarely observed in block copolymers thin films [30], block copolymer solutions [31,32], and surfactants [3335]. It was reported that both FCC and HCP lattice structures appeared as equilibrium structures in a tetrablock copolymer, and the region corresponding to the FCC phase existed outside that corresponding to the $\mathrm{HCP}$ phase in the experimental phase diagram 
[7], which is consistent with our result. Since the radius of spherical micelles used in blend samples did not change irrespective of $\phi_{\text {homo }}$ as described above, the distance between spheres in the case of $\phi_{\text {homo }}=$ $67 \mathrm{wt} \%$ was the longest among the blend samples due to the largest amount of homopolymers. Overlap of neighboring corona chains was smallest in the sample with $\phi_{\text {homo }}=67 \mathrm{wt} \%$. Thus, spherical micelles behaved almost like hard spheres, indicating that the FCC lattice became stable. On the other hand, when the amount of blended homopolymers was low, the corona chains overlapped strongly, and spherical micelles softened. Consequently, it is speculated that the transition from FCC to HCP phase occurred.

\section{Conclusion}

The lattice structures of spherical micelles in PB$\mathrm{PCL} / \mathrm{PB}$ blends were investigated using SAXS. The blend sample with $\phi_{\text {homo }}=40 \mathrm{wt} \%$ showed a cylindrical morphology. In the sample with $\phi_{\text {homo }}=$ $50 \mathrm{wt} \%$, spherical microdomains arrayed into the Frank-Kasper $\sigma$ phase. The HCP lattice structure appeared in the blend with $\phi_{\text {homo }}=56 \mathrm{wt} \%$, whereas the FCC lattice structure was observed for $\phi_{\text {homo }}=$ $67 \mathrm{wt} \%$. Thus, spherical micelles assembled in the following order with increasing $\phi_{\text {homo: }}$ Frank-Kasper $\sigma$, $\mathrm{HCP}$, and FCC phases. In addition, the radius of spheres was almost constant in any type of lattice. The $\sigma$ phase could be observed as a stable phase, because $\varepsilon_{\text {PB.PCL }}(=1.55)$ was slightly larger than the critical value. On the other hand, it is speculated the spherical micelles in the FCC phase behaved like hard spheres such as colloids, leading to stabilization of the FCC lattice. When the distance between spheres was reduced, the corona chains overlapped and softened. Therefore, the FCC lattice should be transformed into the HCP lattice.

\section{Acknowledgements}

This study was partly supported by JSPS KAKENHI Grant Number JP25109008. The SAXS measurements were carried out at the Photon Factory at the High Energy Accelerator Research Organization (approval 2015G092 and 2015G572).

\section{References}

1. F. S. Bates and G. H. Fredrickson, Annu. Rev. Phys.
Chem., 41, 525 (1990).

2. M. W. Matsen and F. S. Bates, Macromolecules, 29, 1091 (1996).

3. Y. Y. Huang, J. Y. Hsu, H. L. Chen and T. Hashimoto, Macromolecules, 40, 1091 (2007).

4. T. Dotera, J. Polym. Sci. Part B, 50, 155 (2012).

5. S. Lee, M. J. Bluemle and F. S Bates, Science, 330, 349 (2010).

6. J. Zhang and F. S. Bates, J. Am. Chem. Soc., 134, 7636 (2012).

7. S. Chanpuriya, K. Kim, J. Zhang, S. Lee, A. Arora, K. D. Dorfman, K. T. Delaney, G. H. Fredrickson and F. S. Bates, ACS Nano, 10, 4961 (2016).

8. S. Lee, C. Leighton and F. S. Bates, Proc. Natl. Acad. Sci. USA, 111, 17723 (2014).

9. T. M. Gillard, S. Lee and F. S. Bates, Proc. Natl. Acad. Sci. USA, 113, 5167 (2016).

10. K. Kim, M. W. Schulze, A. Arora, R. M. Lewis, M. A. Hillmyer, K. D. Dorfman and F. S. Bates, Science, 356, 520 (2017).

11. J. A. Dolan, B. J. Wilts, S. Vignolini, J. J. Baumberg, U. Steiner and T. D. Wilkinson, Adv. Optical Mater., 3, 12 (2015).

12. W. Man, M. Megens, P. J. Steinhardt and P. M. Chaikin, Nature, 436, 993 (2005).

13. J. H. Moon and S. Yang, Chem. Rev, 110, 547 (2010).

14. A. Matsushita and S. Okamoto, Macromolecules, 47, 7169 (2014).

15. A. Noro, Y. Tomita, Y. Matsushita and E. L. Thomas, Macromolecules, 49, 8971 (2016).

16. N. Xie, W. Li, F. Qiu and A. C. Shi, ACS Macro Lett., 3, 906 (2014).

17. M. W. Schultze, R. M. Lewis, J. H. Lettow, R. J. Hickey, T. M. Gillard, M. A. Hillmyer and F. S. Bates, Phys. Rev. Lett., 118, 207801 (2017).

18. H. Takagi, R. Hashimoto, N. Igarashi, S. Kishimoto and K. Yamamoto, J. Phys.: Condens. Matter, 29, 204002 (2017).

19. H. Takagi, N. Igarashi, T. Mori, S. Saijo, H. Ohta, Y. Nagatani, T. Kosuge and N. Shimizu, AIP Conf. Proc., 1741, 030018 (2016).

20. N. Igarashi, N. Shimizu, A. Koyama, T. Mori, H. Ohta, Y. Niwa, H. Nitani, H. Abe, M. Nomura, K. Shioya, K. Tsuchiya and K. Ito, J. Phys.: Conf. Ser., 425, 072016 (2013).

21. T. Hatsui, Synchroton Radiat. News, 27, 20 (2014).

22. S. Choi, K. M. Lee, C. D. Han, N. Sota and T. Hashimoto, Macromolecules, 36, 793 (2003).

23. N. Shimizu, K. Yatabe, Y. Nagatani, S. Saijo, T. Kosuge and N. Igarashi, AIP Conf. Proc., 1741, 
050017 (2016).

24. S. Sakurai, H. Irie, H. Umeda, S. Nomura, H. H. Lee and J. K. Kim, Macromolecules, 31, 336 (1998).

25. S. Förster, A. Timmann, M. Konrad, C. Schellbach, A. Meyer, S. S. Funari, P. Mulvaney and R. Knott, J. Phys. Chem. B, 109, 1347 (2005).

26. L. V. Woodcock, Nature, 385, 141 (1997).

27. S. Auer and D. Frenkel, J. Phys.: Condens. Matter, 14, 7667 (2002).

28. W. Y. Shih, I. A. Aksay and R. kikuchi, J. Chem. Phys., 86, 5127 (1987).

29. L. Athanasopoulou and P. Ziherl, Soft Matter, 13, 1463 (2017).
30. G. E. Stein, E. J. Kramer, X. Li and J. Wang, Macromolecules, 40, 2453 (2007).

31. M. J. Park, J. Bang, T. Harada, K. Char and T. P. Lodge, Macromolecules, 37, 9064 (2004).

32. S. S. Soni, G. Brotons, M. Bellour, T. Narayanan and A. Gibaud, J. Phys. Chem. B, 110, 15157 (2006).

33. X. Zeng, Y. Liu and M. Imperor-Clerc, J. Phys. Chem. B, 111, 5174 (2007).

34. G. C. Shearman, A. I. I. Tyler, N. J. Brooks, R. H. Templer, O. Ces, R. V. Law and J. M. Seddon, $J$. Am. Chem. Soc., 131, 1678 (2009).

35. C. K. Liu and G. G. Warr, Soft Matter, 10, 83 (2014). 